\title{
Elliptic Slotted Antenna with Anisotropic Plasma Dielectric Coating
}

\author{
A-K. Hamid \\ Electrical and Computer Engineering, College of Engineering, University of Sharjah, UAE
}

\begin{abstract}
Radiation properties of axially slotted elliptical antenna embedded in anisotropic plasma material are studied theoretically. The fields are expressed in terms of Fourier series with unknown constants. The radiated field constants are obtained using the boundary value method by invoking the boundary conditions at various surfaces. Results are plotted graphically for the radiation patterns for different geometries and electrical parameters. The results show that the presence of the anisotropic plasma coating has affected the main beam while elliptic antenna beam is less oscillated compared to circular one for the same coating thickness
\end{abstract}

Keywords: axially slotted circular or elliptic antenna, theoretical analysis, anisotropic plasma material, radiation pattern

\section{INTRODUCTION}

Numerous authors have investigated the radiation properties by dielectric coated slotted circular and elliptical cylinders [1-7]. Materials possessing both lossy and lossless metamaterials as well as chiral material have gained considerable attention by several researches [8-10], while possessing anisotropic and anisotropic plasma materials have been studied by [11-14]. The anisotropic plasma material coating is worth to study since the presence of the external magnetic field would make the plasma exhibits anisotropic behavior and its permittivity becomes tensorial form [15-16]. The practical application of antennas with anisotropic plasma dielectric coating it simulates the environment of the space shuttle or missile when enters the earth atmosphere.

The radiation properties produced by slotted elliptical cylinder covered by anisotropic plasma material have not been investigated theoretically. The paper presents theoretical analysis to the radiation by an axially slotted antenna on a conducting elliptic cylinder coated by anisotropic plasma material based on the boundary value method. The presented numerical results will show the effect of anisotropic plasma material coating on the radiation pattern properties of slotted circular or elliptical antenna.

\section{THEORY}

A Consider an infinite conducting elliptic cylinder with axial slot embedded in uniform anisotropic plasma material as shown in Fig.1. The parameters $a_{c}$ and $b_{c}$ represent the inner conducting elliptic cylinder semi-major and semi-minor axes, respectively, while $a$ and $b$ are the semi-major and semi-minor axes of the dielectric anisotropic plasma coating. The antenna angular edges are denoted by $v_{1}$ and $v_{2}$. The elliptical coordinates $(u, v, z)$ may be expressed in terms of Cartesian coordinate systems $(x, y, z)$ as

$$
\begin{aligned}
& x=F \cosh (u) \cos (v) \\
& y=F \sinh (u) \sin (v)
\end{aligned}
$$

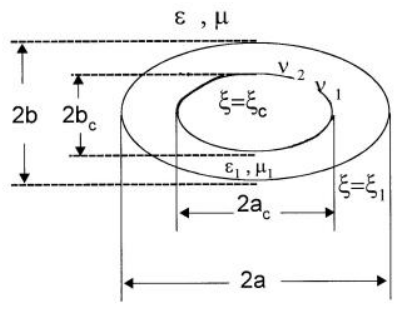

Figure 1: Cross section of Slotted elliptic antenna embedded in uniform anisotropic plasma coating.

where $F$ is the elliptical cylinder semi-focal length. The radiated electric field outside the anisotropic plasma region $\xi>\xi_{1}$ has the form

$E_{z}^{I}=\sum_{m=0}^{\infty} C_{e m} R_{e m}^{(4)}\left(c_{0}, \xi\right) S_{e m}\left(c_{0}, \eta\right)+$

$\sum_{m=1}^{\infty} C_{o m} R_{o m}^{(4)}\left(c_{0}, \xi\right) S_{o m}\left(c_{0}, \eta\right)$

where $C_{e m}$ are arbitrary radiated field constants to be determined later, $S_{e m}$ are the even and odd angular Mathieu functions of order m, while $R_{e m}^{(4)}$ are the even and odd radial Mathieu functions of the fourth kind, $\xi=\cosh u, \eta=\cos v, c_{0}=k F$, and $k=\omega \sqrt{\mu \varepsilon}$.

Assuming the anisotropic plasma region has permeability equals to free space and permittivity tensor defined as [8]

$$
[\varepsilon]=\left[\begin{array}{ccc}
\varepsilon_{1} & j \varepsilon_{2} & 0 \\
-j \varepsilon_{2} & \varepsilon_{1} & 0 \\
0 & 0 & \varepsilon_{3}
\end{array}\right]
$$

where $\varepsilon_{1}, \varepsilon_{2}$ and $\varepsilon_{3}$ are complex functions of $\omega$, electron density, collision frequency, and the external magnetic field. 
Similarly, the electric field inside the anisotropic plasma dielectric coating $\xi_{c}<\xi<\xi_{1}$ has the form

$$
\begin{aligned}
E_{z}^{I I}= & \sum_{m=0}^{\infty}\left[A_{e m} R_{e p}^{(1)}\left(c_{1}, \xi\right)+B_{e m} R_{e p}^{(2)}\left(c_{1}, \xi\right)\right] S_{e m}\left(c_{1}, \eta\right)+ \\
& \sum_{m=1}^{\infty}\left[A_{o m} R_{o p}^{(1)}\left(c_{1}, \xi\right)+B_{o m} R_{o m}^{(2)}\left(c_{1}, \xi\right)\right] S_{o m}\left(c_{1}, \eta\right)
\end{aligned}
$$

where $c_{1}=k_{1} F, \quad k_{1}=k / \sqrt{m}, \quad m=\varepsilon_{1} /\left(\varepsilon_{1}^{2}-\varepsilon_{2}^{2}\right)$, $A_{\substack{e m \\ o m}}$ and $B_{\substack{e m \\ o m}}$ are arbitrary constants, $R_{\substack{e p \\ o p}}^{(1)}$ and $R_{\substack{e p \\ o p}}^{(2)}$ are the radial Mathieu functions of the first and second kind. The magnetic field components in regions (I) and (II) are derived using Maxwell's equations and expressed as

$$
\begin{aligned}
& H_{v}^{I}=\frac{-j}{Z k h}\left\{\begin{array}{c}
\sum_{m=0}^{\infty} C_{e m} R_{e m}^{\prime(4)}\left(c_{0}, \xi\right) S_{e m}\left(c_{0}, \eta\right)+ \\
\sum_{m=1}^{\infty} C_{o m} R_{o m}^{\prime(4)}\left(c_{0}, \xi\right) S_{o m}\left(c_{0}, \eta\right)
\end{array}\right\} \\
& H_{v}^{I I}=\frac{-j}{Z_{c} k_{1} h}\left\{\sum_{m=0}^{\infty}\left[A_{e m} R_{e m}^{\prime(1)}\left(c_{1}, \xi\right)+B_{e m} R_{e m}^{\prime(2)}\left(c_{1}, \xi\right)\right] S_{e m}\left(c_{1}, \eta\right)\right. \\
& \left.+\sum_{m=1}^{\infty}\left[A_{o m} R_{o m}^{\prime(1)}\left(c_{1}, \xi\right)+B_{o m} R_{o m}^{\prime(2)}\left(c_{1}, \xi\right)\right] S_{o m}\left(c_{1}, \eta\right)\right\}
\end{aligned}
$$

$$
\begin{aligned}
& \text { integrating over } 0<v<2 \pi \text {, we obtain }
\end{aligned}
$$

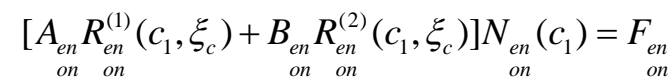

$$
\begin{aligned}
& =\int_{v_{1}}^{v_{2}} F(v) S_{\substack{e n \\
o n}}\left(c_{1}, \eta\right) d v
\end{aligned}
$$

The $\mathrm{z}$ axial component of the electric field on the cylinder surface $\left(\xi=\xi_{c}\right)$ must be zero except between the slot edges. This leads to

$\sum_{m=0}^{\infty}\left[A_{e m} R_{e p}^{(1)}\left(c_{1}, \xi_{c}\right)+B_{e m} R_{e p}^{(2)}\left(c_{1}, \xi_{c}\right)\right] S_{e m}\left(c_{1}, \eta\right)+$ $\sum_{m=1}^{\infty}\left[A_{o m} R_{o p}^{(1)}\left(c_{1}, \xi_{c}\right)+B_{o m} R_{o m}^{(2)}\left(c_{1}, \xi_{c}\right)\right] S_{o m}\left(c_{1}, \eta\right)=$ $F(v) \quad v_{1}<v<v_{2}$

$0, \quad$ elsewhere.

Multiplying both sides of (12) by $S_{\substack{e n \\ o n}}\left(c_{1}, \eta\right)$ and

where $h=F \sqrt{\left(\cosh ^{2} u-\cos ^{2} v\right.}, Z$ is the characteristic wave impedance in free space while $Z_{c}$ is the wave impedance of the anisotropic plasma medium and given by $Z_{c}=\sqrt{m} Z$. The prime in equations (6) and (7) indicates derivative with respect to $u$.

The $\mathrm{z}$ axial component of the electric field should to be continuous across the anisotropic plasma layer at $\xi=\xi_{1}$, this leads to

$$
\begin{aligned}
& \left.\underset{\substack{e n \\
o n}}{A_{e n} R_{o n}^{(1)}}\left(c_{1}, \xi_{1}\right)+B_{\substack{e n \\
o n}} R_{\substack{e n \\
o n}}^{(2)}\left(c_{1}, \xi_{1}\right)\right] N_{\substack{e n \\
o n}}\left(c_{1}\right)= \\
& \sum_{m=0}^{\infty} C_{\substack{e m \\
o m}} R_{e m}^{(4)}\left(c_{0}, \xi_{1}\right) M_{\substack{e n m \\
o m n}}\left(c_{1}, c_{0}\right)
\end{aligned}
$$

where

$$
\begin{aligned}
& N_{\substack{e n \\
o n}}\left(c_{1}\right)=\int_{0}^{2 \pi}\left[S_{\substack{e n \\
o n}}\left(c_{1}, \eta\right)\right]^{2} d v \\
& M_{\substack{e n m \\
o m n}}\left(c_{1}, c_{0}\right)=\int_{0}^{2 \pi} S_{\substack{e n \\
o n}}\left(c_{1}, \eta\right) S_{\substack{e m \\
o n}}\left(c_{0}, \eta\right) d v
\end{aligned}
$$

in equations (13) may be computed as [4]

$$
\begin{aligned}
& F(v)=E_{0} \cos \left[\pi\left(v_{0}-v\right) /(2 \alpha)\right] \\
& v_{0}=\left(v_{1}+v_{2}\right) / 2 \\
& \alpha=\left(v_{2}-v_{1}\right) / 2
\end{aligned}
$$

The even and odd angular Mathieu functions are written in terms of Fourier series as

$$
\begin{aligned}
& S_{\text {en }}\left(c_{1}, \eta\right)=\sum_{k} D_{e}^{k}\left(c_{1}, n\right) \cos (k v) \\
& S_{\text {on }}\left(c_{1}, \eta\right)=\sum_{k} D_{o}^{k}\left(c_{1}, n\right) \sin (k v)
\end{aligned}
$$

Substituting equations (14)-(18) into equation (13), $F_{e n}$ and $F_{o n}$ can finally be written in terms of the slot field as

$$
F_{e n}=E_{o} \sum_{k} D_{e}^{k}\left(c_{1}, n\right) \int_{v_{1}}^{v_{2}} \cos \left[\pi\left(v-v_{0}\right) /(2 \alpha)\right] \cos (k v) d v
$$

$$
F_{o n}=E_{o} \sum_{k} D_{o}^{k}\left(c_{1}, n\right) \int_{v_{1}}^{v_{2}} \cos \left[\pi\left(v-v_{0}\right) /(2 \alpha)\right] \sin (k v) d v
$$

(10) Solving for the constants $B_{e n}$ from equation (13) and The $v$ angular component of the magnetic field at $\xi=\xi_{1}$ incorporating the result in equations (8) and (11) by should also be continues and require that

eliminating $A_{\substack{e n \\ o n}}$, yields the radiated field constants $C_{\substack{e n \\ o n}}$. 


\section{III.NUMERICAL RESULTS}

After computing the radiated field constants $C_{\substack{e n \\ \text { on }}}$, the farfield radiation pattern, antenna gain, and the aperture conductance may be evaluated. The slotted antenna gain is expressed as [4]

$$
G(\varphi)=\frac{1}{Z_{0} k \rho}\left[\begin{array}{l}
\left|\sum_{n=0}^{\infty} j^{n} C_{e n} S_{e n}\left(c_{0}, \cos \varphi\right)\right|^{2}+ \\
\left|\sum_{n=1}^{\infty} j^{n} C_{o n} S_{o n}\left(c_{0}, \cos \varphi\right)\right|^{2}
\end{array}\right]
$$

The accuracy of the numerical results is checked against published data for slotted circular or elliptic antenna embedded in dielectric material. The slotted antenna cross section parameters used for comparison are taken to be $a_{c}=\lambda, b_{c}=\lambda / 2, b=b_{c}+t$, while $t$ is the thickness of the anisotropic plasma layer, $v_{0}=90^{\circ}$ and $\alpha=2.8657^{\circ}$. Figure 2 shows the radiation pattern (solid line) plotted against the angle $\varphi$ for $\varepsilon_{1}=4, \varepsilon_{2}=j 0, \varepsilon_{3}=4$ and $\mu_{r}=1$, and $t=0.25 \lambda$ to check the result accuracy [4]. Both results are in full agreement. It also shows the effect of increasing $\varepsilon_{2}$ to $j 0.5$ and $j 1.0$ where we can see there is significant change at the antenna location especially for the case of $\varepsilon_{2}=j 1.0$ and minor change at other locations. Figure 3 shows the same case but for circular antenna. It can be seen that the circular antenna is affected by the anisotropic plasma coating at all angles and the pattern oscillates significantly than that the elliptic antenna case. Figure 4 is similar to Figure 2 except for $\varepsilon_{1}=5$. Figure 5 is similar to Figure 4 except for several thicknesses and $\varepsilon_{2}=j 0.5$

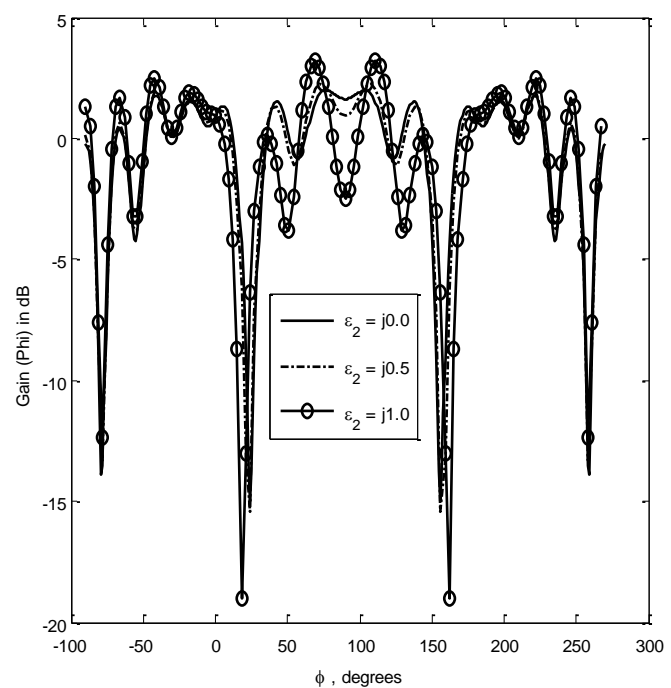

Figure 2: Radiation pattern of elliptic antenna covered by anisotropic plasma material with $\varepsilon_{1}=4.0, \varepsilon_{3}=4.0$, $\mu_{r 1}$ $=1.0, a_{c}=\lambda, b_{c}=\lambda / 2, b=b_{c}+0.25 \lambda$.

Copyright to IJARCCE

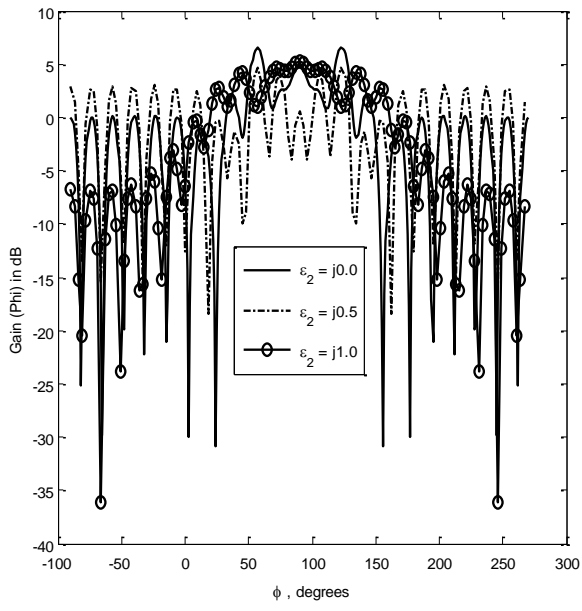

Figure 3: Radiation pattern of circular antenna covered by anisotropic plasma material with $\varepsilon_{1}=4.0, \varepsilon_{3}=4.0, \mu_{r 1}$ $=1.0, a_{c}=\lambda, b_{c}=\lambda, b=b_{c}+0.25 \lambda$.

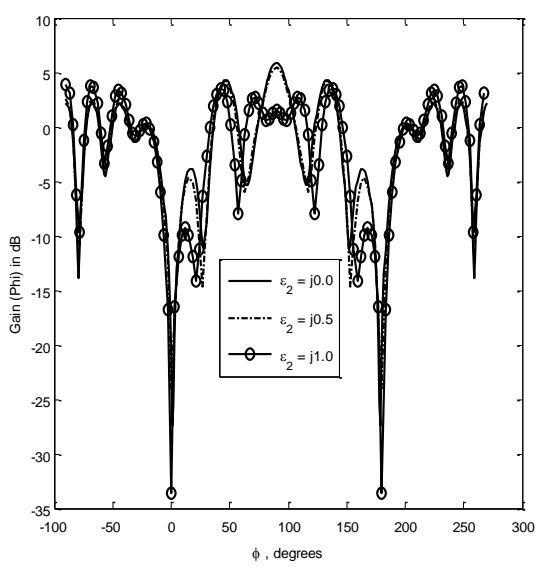

Figure 4: Radiation pattern of elliptic antenna covered by anisotropic plasma material with $\varepsilon_{1}=5.0, \varepsilon_{3}=4.0, \mu_{r 1}$ $=1.0, a_{c}=\lambda, b_{c}=\lambda / 2, b=b_{c}+0.25 \lambda$.
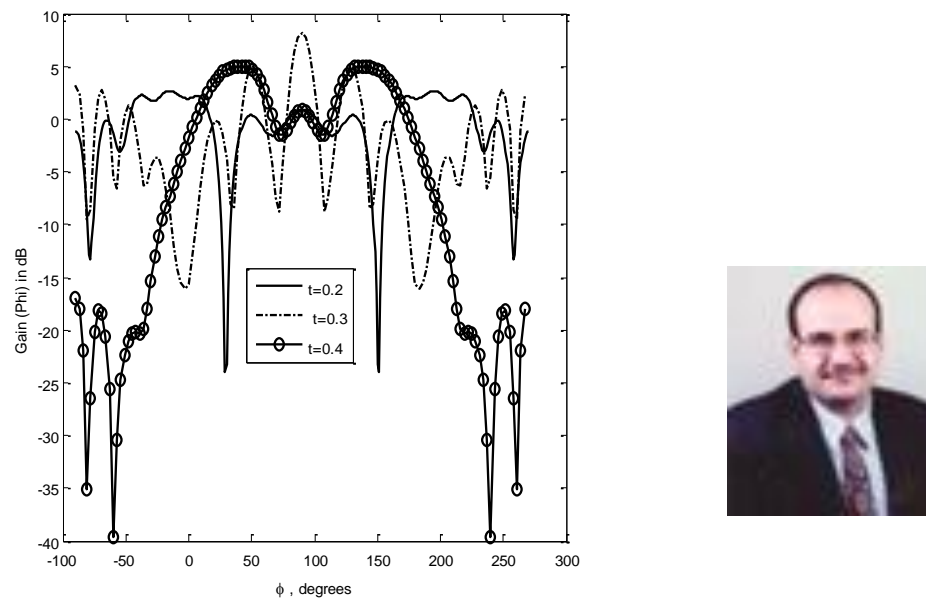

Figure 5: Radiation pattern of elliptic antenna covered by anisotropic plasma material with $\varepsilon_{1}=5.0, \varepsilon_{2}=j 0.5$, $\varepsilon_{3}=4.0, \mu_{r 1}=1.0, a_{c}=\lambda, b_{c}=\lambda / 2, b=b_{c}+t$. 


\section{IV.CONCLUSION}

The The radiation properties of axially slotted circular or elliptical antenna covered by uniform anisotropic plasma material were studied theoretically. It was shown that the presence of anisotropic plasma material layer change the radiation pattern of the antenna especially at the antenna location for moderate thicknesses. Figure 5 show that the antenna main beam may be enhanced for some specific parameters. Further, the coated slotted elliptic antenna was less oscillated by the presence of the same thickness of anisotropic material when compared to the circular antenna.

\section{ACKNOWLEDGMENT}

The author appreciates the support provided by the University of Sharjah, United Arab Emirates

\section{REFERENCES}

[1] L. Shafai, "Radiation from an axial slot antenna coated with a homogenous material," Canadian J. Phys., vol. 50, no. 23, 1972.

[2] J. H. Richmond, "Axial slot antenna on dielectric coated elliptic cylinder,” IEEE Trans. Antennas Propagat., vol. AP-37, pp. 12351241, Oct. 1989.

[3] H. A. Ragheb, A. Sebak, and L. Shafai, "Radiation by axial slots on dielectric coated nonconfocal conducting elliptic cylinder," IEE Proc. Microw. Antennas Propagat., vol. 143, no. 2, pp. 124-130, April 1996.

[4] M.I. Hussein, and A-K. Hamid, "Radiation characteristics of N axial slots on a conducting elliptical antenna coated by a lossy dielectric layer," Canadian Journal of Physics, vol. 82, no. 2, pp. 141-149, 2004.

[5] B. N. Khatir, M. Al-Kanhal, and A. Sebak, Electromagnetic wave scattering by elliptic chiral cylinder," Journal of Electro-magnetic Waves and Applications, Vol. 20, No. 10, pp. 1377-1390, 2006.

[6] B. N. Khatir and A. R. Sebak , "Slot Antenna on a Conducting Elliptic Cylinder Coated by Chiral Media," Electromagnetics, Vol 29, pp $522-540,2009$

[7] H.C. Chen and D.K. Cheng, "Scattering of Electromagentic by anisotropic plasma coated conducting cylinder," IEEE Trans. on Antennas and Propagation, Vol. AP-12, pp. 348-353, 1964.

[8] H.C. Chen and D.K. Cheng, "Radiation from an axially slotted anisotropic plasam claded cylinder," IEEE Trans. on Antennas and Propagation, Vol. AP-13, pp. 395-401, 1965.

[9] X.-B. Wu and W. Ren , "Axial slot antenna on an anisotropic dielectric-coated circular cylinder," IEE Proceedings - Microwaves, Antennas and Propagation, vol. 141, Issue 6, p. 527 - 530, 1994.

[10] A. Gaffar, M.Z. Yaqoob, M. Alkanhal, M. Sharif and Q.A. Naqvi, "Electromagntic scttering from anistropic plasma coated perfect electromagntic conductor cylinders," Internationa Journal of Electronics and Commuinactions (AEU), vol. 86, no.8, pp. 767-772, 2014.

\section{BIOGRAPHY}

A-K. Hamid was born in Tulkarm, West Bank, on Sept. 9, 1963. He received the B.Sc. degree in Electrical Engineering from West Virginia Tech, West Virginia, U.S.A. in 1985. He received the M.Sc. and Ph.D. degrees from the University of Manitoba, Winnipeg, Manitoba, Canada in 1988 and 1991, respectively, all in Electrical Engineering. From 1991-1993, he was with Quantic Laboratories Inc., Winnipeg, Manitoba, Canada, developing two and three dimensional electromagnetic field solvers using boundary integral method. From 19942000 he was with the faculty of electrical engineering at King Fahd University of Petroleum and Minerals, Dhahran, Saudi Arabia. Since Sept. 2000 he has been an associate Prof. in the electrical/electronics and computer engineering department at the University of Sharjah,
Sharjah, United Arab Emirates. His research interest includes EM wave scattering from two and three dimensional bodies, propagation along waveguides with discontinuities, FDTD simulation of cellular phones, and inverse scattering using neural networks. 